\section{Paper Partition Chromatography of Sulphonamides}

'I'HE recent communications on paper partition chromatography of sulphonamides by R. Robinson ${ }^{1}$ and A. E. Steel ${ }^{2}$ prompt us to direct attention to earlier reports by W. H. l.ongenecker ${ }^{3}$ and especially by G. Thomas et al.4. Isolated examples of the use of this method in the analysis of sulphonamides can also be found in the work of B. Ekman ${ }^{5}$ and H. ( $A$. Bray et al. ${ }^{6}$.

We have used several solvent systems, including the butanol-ammonia mixture proposed by $R$. ( $\dot{x}$. Shepherd ${ }^{3}$. Our results agree with those reported by R. Robinson ${ }^{1}$, although the movement of sulphacetamide was found to be much slower.

For the detection of the sulphonamide spots by the diazo and coupling reaction, we found diazotization by gaseous nitrogen oxides very useful. The strips were hung for 30 sec. in a glass vessel at the bottom of which a few millilitres of diluted sulphuric acid had been added to sodium nitrite.

The detection of sulphonamides lacking an unsubstituted aromatic amino group offers difficulty, as almost every colour reaction of the sulphonamides is due to the presence of this group. Succinylsulphathiazole, phthalylsulphathiazole and phthalylsulphacetamide could be detected, however, by the use of an indicator spray, because they are carboxylic acids.

For quantitative measurements we prefer the use of Ehrlich's reagent. After detection by spraying with a 1 per cent solution of $p$-dimethylamino benzaldehyde in diluted hydrochloric acid, the spots were separately eluted with an acid-buffered solution of the same reagent for determination of the sulphonamide content by a modification of the procedure of C..T. O. Morris ${ }^{\imath}$.

(iO LAM SAN

A. J. ULTÉE, JUN.

c/o Fibre Research Institute T.X.().

$$
\text { Delft. }
$$

Niture, 168, 512 (1951).

Nature, 168, 877 (1951).

Anat. Chem., 21, $1402(1949)$

J. Pharm. Belg., (N.S.), 5, 26:3 (1950).

Acte Chim. Scand., 2, 383 (1948).

"Biochem. J., 48, 400 (1951).

Binchem. J. 35, 952 (1941).

\section{Inhibitory Effect of Tetraethylammonium- bromide in Alloxan Diabetes}

I'HE mechanism of the effect of alloxan has been examined thoroughly by several investigators. For example, Abderhalden ${ }^{1}$ found that if the application of alloxan was preceded by an injection of cysteine, he was able to prevent diabetes. Brickmann and Wertheimer: found that the level of reduced glutathione in the blood fell after an injection of alloxan. In our previous work ${ }^{3}$, we showed that alloxan reacts with SH-groups of blood proteins; this was proved polarographically. Further, we found that we could trace the fall of reduced glutathione not only in the blood but also, after a longer period of time following the injection of alloxan, in the pancreas, liver and, mainly, in the adrenals ${ }^{4}$.

Jimenez-Diaz, Grande-Covian and De Oya ${ }^{5}$ demonstrated for the first time that tying up the renal hilus prevents alloxan diabetes. Houssay" states that alloxan diabetes should not be regarded as concerned entirely with the kidneys, as would seem from previous work ; for example, a bilateral nephrectomy causes alloxan diabetes. Houssay and Martínez ${ }^{7}$ analysed thoroughly these findings and found that it was sufficient to block the nerve ductus in the renal hilus to prevent diabetes. Houssay et al. explained these phenomena with reference to the motorvascular system of the pancreas. These views have been criticized; for example, A. Gold ${ }^{8}$ found no significant differences in rats the kidneys of which were tied up before injecting alloxan, when compared with normal rats; J. L. Duff, D. C. Wilson and $\mathrm{G}$. C. MeMillan ${ }^{9}$ also did not succeed in reproducing these results with rabbits.

We have therefore endeavoured to find out whether, after tying up the renal hilus, the typical changes in glutathione metabolism would occur following an injection of alloxan. A group of rats starved for $24 \mathrm{hr}$. was ancesthetized with ether. By the lumbral route both kidneys were uncovered and the renal hilus was tied up. Five hours later, alloxan was injected subcutaneously into the rats in doses of $15 \mathrm{mgm}$. $100 \mathrm{gm}$. body-weight. The rats were killed with blows on the head after the following intervals : 15 min., 45 min., 90 min., 300 min. We used anhydrous alloxan (Roche, Basle). The livers and pancreas were analysed by the modified Kühnau method to determine reduced glutathione; compared with a group of animals the kidneys of which were not tied up, the level of reduced glutathione had not fallen as it does typically in normal animals injected with alloxan.

With another group of animals we blocked the sympathetic ganglions with tetraethylammoniumbromide, which was injected subcutaneously in doses of $3 \mathrm{mgm}$./ $1 \mathrm{kgm}$. body weight. It proved later that thcse doses were quite sufficient for our purposes. Five minutes after the injection of tetraethylammoniumbromide, we injected alloxan subcutaneously into the animals in the same doses as in the previous experiments. The rats were killed at definite intervals, and the reduced glutathione in their livers, pancreas and adrenals determined quantitatively. It was found that after tetraethylammoniumbromide injections, there was no fall in the level of reduced glutathione following the injection of alloxan.

This experiment was repeated, except that after the injection of alloxan the rats were put into metabolism cages, where they were given water and food ad libitum. Then we used the Cole method for determining glucose in urine to detect glycosuria. After a few days the rats were killed and their blood glucose determined by the Hagedorn-Jensen method.

This experiment was carried out with five subgroups of eight rats each. Tetraethylammoniumbromide was injected in the same doses as in the previous case; the doses of alloxan were also identical. In individual subgroups except group 1 , which served as control, we traced the dependence of the origin and character of diabetes upon the length of time which passed between the injection of tetraethylammoniumbromide and the introduction of alloxan :

(a) Rats of one subgroup were given alloxan only. They all developed polyuria and glycosuria, and during the experiment they were steadily losing weight. After death the value of blood sugar averaged $212 \mathrm{mgm}$. per cent.

(b) Alloxan was injected simultaneously with the injection of tetraethylammoniumbromide. Glycos- 